\section{Keine Aussagekraft bei Verdacht auf Lungenembolie}

Beim klinischen Verdacht auf eine akute Lungenembolie erlaubt die Multidetektor-Computertomografie (MDCT) keine validen

Aussagen. Eine hiermit diagnostizierte Rechtsherzbelastung lässt sich in der Echokardiografie nicht sicher reproduzieren. Auch der klinische Verlauf korreliert nicht mit der MDCTVerdachtsdiagnose auf eine akute Lungenembolie, wie eine prospektive Studie jetzt belegte. Thorax 2014; 69: 109-115

Beim klinischen Verdacht auf Lungenembolie ist eine zeitnahe Diagnostik essenziell. Die Echokardiografie gilt hier als klinischer Standard. Aufgrund der benötigten Expertise steht dieses Verfahren nicht immer zeitnah zur Verfügung. Hier bietet sich das MDCT als Alternative an. Vereinfachte Algorithmen erlauben es, analog zur Echokardiografie, das Volumen des rechten Ventrikels zu berechnen und so die emboliebedingte Rechtsherzinsuffizienz zu erkennen. Die Autoren setzten das MDCT bei 848 Patienten mit Verdacht auf akute Lungenembolie zur primären Not- falldiagnostik ein. Rekrutiert wurden nur normotensive Patienten. Spätestens nach 24 Stunden erfolgte eine Echokardiografie. Die Laborparameter Troponin und BNP ergänzten die Diagnostik.

Die Autoren verfolgten für 30 Tage den klinischen Verlauf mit besonderem Fokus auf hämodynamische Instabilitäten. Primär prüften sie, ob ein positiver MDCT-Befund mit der Mortalitätsprognose korrelierte. Sekundäres Studienziel war die Frage, ob eine im MDCT erkannte, rechtsventrikuläre Dilatation, auf einen komplizierten Ver- lauf mit hämodynamischen Komplikationen hinweist. Bei 63\% der Patienten diagnostizierte das MDCT eine akute Dilatation des rechten Ventrikels. Die durchschnittliche Kurzzeitmortalität von 4,5\% zeigte keinen Zusammenhang mit der im MDCT ermittelten rechtsventrikulären Situation. Der MDCT-Befund korrelierte auch nicht mit der Rate der hämodynamischen Komplikationen. Auffallend war allerdings, dass Patienten mit positivem MDCT erhöhte Troponin- ( 0,10 vs. $0,03 \mathrm{ng} / \mathrm{ml})$ und BNPWerte (269 vs. $180 \mathrm{pg} / \mathrm{ml}$ ) aufwiesen. Die echokardiografische Überprüfung konnte den MDCT-Befund nur in 31\% der Fälle verifizieren. Patienten mit negativem MDCTBefund dagegen zeigten zu 9\% echokardiografisch eine rechtsventrikuläre Dilatation.

\section{Fazit}

Beim klinischen Verdacht auf Lungenembolie könne nicht auf das MDCT zur Verdachtsabklärung zurückgegriffen werden. Eine so radiologisch ermittelte, rechtsventrikuläre Dilatation lässt sich i.d.R. echokardiografisch nicht verifizieren und korrespondiert nicht mit dem für die Lungenembolie typischen klinischen Verlauf. Die Autoren raten deshalb davon ab, aus einem bei dieser Fragestellung erhobenen MDCT-Befund therapeutische Konsequenzen abzuleiten.

\section{Dr. Horst Gross, Berlin}

Viren konnte gezeigt werden, dass sich das neu entdeckte Virus zwar gut in menschlichen Zellen vermehrt, in Mäusen aber zu keiner Erkrankung führt. Ebenso wichtig ist der Nachweis, dass die Virusgenome so stark voneinander abweichen, dass sie sich mit Genen menschenpathogener Influenza-A-Viren nicht mischen können. Somit stellt das H17N10-Virus sehr wahrscheinlich kein Gefahrenpotenzial für die Entstehung einer neuen hochinfektiösen Virusvariante dar. „Unsere Ergebnisse schließen zwar eine Möglichkeit der Übertragung des Fledermaus-Influenza-Virus auf Menschen nicht völlig aus, aber die Gefahr, die von diesen H17N10Viren ausgeht, scheint doch vergleichsweise gering zu sein“, erklärt Prof. Schwemmle. Ein H17N10-ähnliches Virus wurde kürzlich aus Fledermäusen in Peru isoliert. Ob noch weitere Influenzaviren in Fledermäusen zirkulieren bleibt abzuwarten.

Nach einer Mitteilung des Universitätsklinikums Freiburg 